\title{
A Multiagent System for Microgrid Operation in the Grid-interconnected Mode
}

\author{
Hak-Man Kim ${ }^{\dagger}$ and Tetsuo Kinoshita*
}

\begin{abstract}
This paper presents a multiagent system for microgrid operation in the grid-interconnected mode. An energy market environment with generation competition is adopted for microgrid operation in order to guarantee autonomous participation and meet the requirements of participants in the microgrid. The modified Contract Net Protocol (CNP) is used as a protocol for interactions among agents. The multiagent system for microgrid operation based on the modified CNP and the energy market environment is designed and implemented. To verify the feasibility of the suggested multiagent system, experiments on three operation conditions are carried out.
\end{abstract}

Keywords: Microgrid, Microgrid operation, Multiagent system, Modified Contract Net Protocol, Cooperative multiagent system

\section{Introduction}

A microgrid composed of distribution generators (DGs), storage devices, and loads is a small-scale power grid and is evaluated as a green energy system. To commercialize a microgrid, many research, development, and demonstration projects have been undertaken in many countries [1], [2]. In the near future, it is expect that many microgrids will be connected to distribution systems.

In recent years, big changes have taken place such as the introduction of competition and an increase in distributed generators according to the Kyoto protocol on climate change in power systems. As a result of these changes, it is expected that the direction of power flow in a horizontal power system will no longer be predictable and that this will affect the existing protection and operation schemes of power systems. Because of these changes, increased attention has recently been placed on autonomous power systems in the field of power engineering [3]. In particular, to implement autonomous systems, intelligent agent technologies have been studied because of the following characteristics of the agent: reactivity, pro-activeness, and social ability [4].

An intelligent agent, which is simply called an agent, can perceive its environment, make a decision against environmental changes, and can act to resolve them autonomously according to its designed purpose. Furthermore, it is well-known that this agent is effective in distributed systems or problems [4]. The multiagent system is a system comprising of intelligent agents that interact to solve given problems in the multiagent system. In power engineering, the multiagent system has been studied for various tasks

$\bar{\dagger}$ Corresponding Author: Dept. of Electrical Engineering, University of Incheon, Incheon, Korea. (hmkim@incheon.ac.kr)

* Research Institute of Electrical Communication, Graduate School of Information Science, Tohoku University, Sendai, Japan. (kino@riec.tohoku.ac.kr)

Received: January 26, 2010; Accepted: March 24, 2010 such as monitoring transformer condition [5], system restoration [6], and dispatching of distributed generators [7]. The multiagent system can be designed as a cooperative model or a competitive model. In the cooperative multiagent system, agents collaborate to solve a common problem. In the competitive multiagent system, agents compete to solve the given problem for their own profit. Recently, for microgrid control, a multiagent system based on an auction algorithm of an energy auction was studied by Dimeas and his colleague [8].

The Contract Net Protocol (CNP) was developed to solve cooperative distributed problems in communications [9]. The CNP has been used as a protocol for interactions among cooperative agents. The basic procedure of the CNP is composed of an announcement of a new task, bid, and awarding contracts. The CNP has become the most implemented and extensively studied framework for distributed problem solving in spite of its simplicity [4].

In this paper, we propose a multiagent system for microgrid operation in the grid-interconnected mode. The CNP is appropriately modified for microgrid operation. The modified CNP is used as a protocol for interactions among agents. An energy market environment with a generation competition instead of an energy auction as the initial model of an energy market for microgrid operation is adopted to meet the requirements of participants in the microgrid, which will be described in Section 2.1. Final suppliers as contractors of the modified CNP are decided by merit order [10], which is a classical algorithm for economic dispatch. The multiagent system based on the modified CNP and the energy market environment is designed and implemented to solve the operation problem of the microgrid. To evaluate the feasibility of the suggested multiagent system for microgrid operation in the gridinterconnected mode, three experiments are carried out. The experiment results are compared with the results of linear programming for discussion. 


\section{Agent-based Microgrid Operation on Energy Market Environment}

\subsection{Microgrid Operation on Energy Market Envi- ronment with Generation Competition}

Fig. 1 shows the microgrid configuration, where the Microgrid Operation and Control Center (MGOCC) has important functions such as operating the microgrid, controlling and monitoring protection devices, and communicating with participants in the microgrid and power grid. The MGOCC is sometimes called the Microgrid Control Center (MGCC) [8].

To be able to maintain a constant frequency such as 50 $\mathrm{Hz}$ or $60 \mathrm{~Hz}$ is an important requirement for microgrid operation. For this, in the grid-interconnected mode, microgrid operation is required to transmit power to or receive power from the connected power grid according to supply surplus or supply shortage.

The following shows the basic requirements of participants including suppliers and consumers of the microgrid:

(R1) Power suppliers want to supply power at a high price.

(R2) On the other hand, power consumers hope to use power at a low price.

(R3) Their autonomous participation should be guaranteed.

To guarantee the basic requirements for microgrid operation, introducing an energy market environment is a good choice. In this paper, we introduce an energy market environment with generation competition instead of an energy auction as the initial model of an energy market for microgrid operation.

In this study, it is assumed that at the beginning of an operation interval, from the power grid the MGOCC receives information on two power transaction prices for the next interval: the selling price $\left(P_{S}\right)$ for selling power to the power grid and the buying price $\left(P_{B}\right)$ for buying power from the power grid. The MGOCC should establish an operation plan for the next interval and should implement an operation plan established during the previous interval as shown in Fig. 2 like the operation of power grids. In general markets, the selling price to the market is generally lower than the buying price from the market. In this study, it is

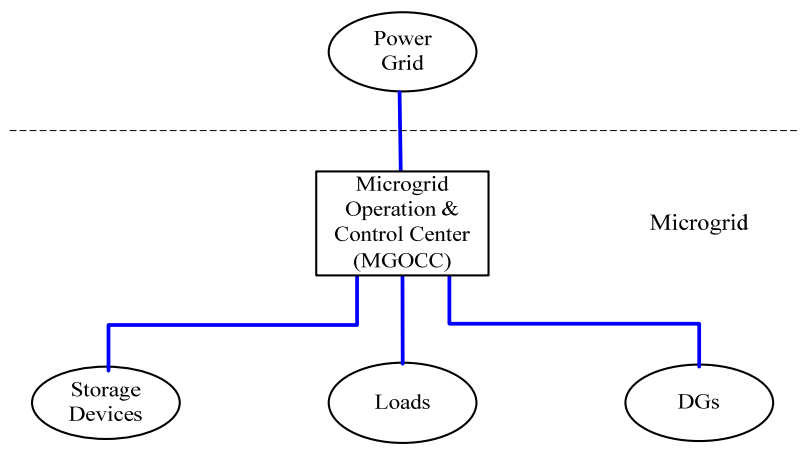

Fig. 1. Microgrid configuration [11].

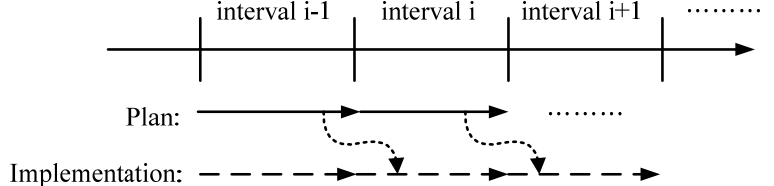

Fig. 2. Microgrid operation procedure [11].

assumed that $P_{S}$ is lower than $P_{B}$, as is the case in general markets. The length of an interval and detailed procedures depend on the energy market rules that are enforced.

The following shows the operation rules of the adopted energy market for microgrid operation:

- At the beginning of an interval, the MGOCC receives $P_{S}$ and $P_{B}$ for the next interval from the power grid.

- After receiving two transaction prices $\left(P_{S}\right.$ and $\left.P_{B}\right)$, the MGOCC announces the prices to every participant.

- Each consumer should inform the MGOCC of its power demand for the next interval.

- Each supplier whose supply price is lower than or equal to $P_{B}$ can inform the MGOCC of its intention to supply power with a bid price and a supply amount for the next interval.

- The MGOCC selects final suppliers by descending order of production costs (merit order).

- Surplus power of suppliers not selected by merit order is sold to the power grid, and shortage power required to compensate for any supply shortage is purchased from the power grid.

- The market clearing price (MCP) of the Microgrid $\left(M C P_{M G}\right)$ is decided as the maximum bid price of the final suppliers. When the maximum bid price is lower than $P_{S}$, the $M C P_{M G}$ is replaced by $\mathrm{P}_{\mathrm{S}}$ in order to satisfy the requirements of suppliers $(R 2)$.

According to the above market rules, the $M C P_{M G}$ always exists between $P_{S}$ and $P_{B}$ as follows:

$$
P_{S} \leq M C P_{M G} \leq P_{B}
$$

Therefore, by adopting the energy market environment with generation competition for Microgrid operation, the requirements of all participants $(R 1, R 2$, and $R 3)$ are satisfied as follows:

- Power suppliers can supply power at a price higher than or equal to $P_{S}$.

- Power suppliers not selected by merit order among bidders whose bid prices are lower than or equal to $P_{S}$ also can sell power to the power grid at $P_{S}$.

- On the other hand, power consumers can purchase power at a price lower than or equal to $P_{B}$.

- The autonomous participation of all participants is guaranteed by adopting the energy market environment.

The existing profit difference among power suppliers in microgrids will ultimately promote the development and use of production units with lower production costs. 


\subsection{Agent-based Microgrid Operation}

An agent has the following capabilities: reactivity, proactiveness, social ability, and so on [4]. And it is able to sense the external environment, effectively make a decision based on design purpose against the environment and act according to the decision.

The agent is modeled by the following mathematical expressions [4], [12]. The set of the environment states $(E)$ is expressed as follows:

$$
E=\left\{e_{0}, e_{1}, e_{2}, \ldots, e_{n}\right\}
$$

where $e_{0}$ is an initial environment state. The set of possible actions of the agent against each environment state $(A)$ is expressed as follows:

$$
A=\left\{a_{1}, a_{2}, a_{3}, \ldots, a_{n}\right\}
$$

An agent $(\mathrm{Ag})$ can be expressed as a function of environment states and actions as follows:

$$
A g: E \rightarrow A
$$

A run of an agent in an environment $(r)$ is a sequence of interleaved environment states and actions as follows:

$$
r: e_{0} \stackrel{a_{1}}{\longrightarrow} e_{1} \stackrel{a_{2}}{\longrightarrow} e_{2} \stackrel{a_{3}}{\longrightarrow} \ldots \stackrel{a_{n-1}}{\longrightarrow} e_{n}
$$

To construct a multiagent system, the agent communication language (ACL) and knowledge sharing among agents are required [4], [13]. The CNP is the most used protocol for interactions among agents [4]. The main process of the CNP involves the following basic steps [12]:

Step 1: A manager announces the existence of a task via a broadcast message.

Step 2: Agents evaluate the announcement and capable agents submit bids.

Step 3: The manager awards a contract to the most suitable agent among bidding agents as a contractor for the task.

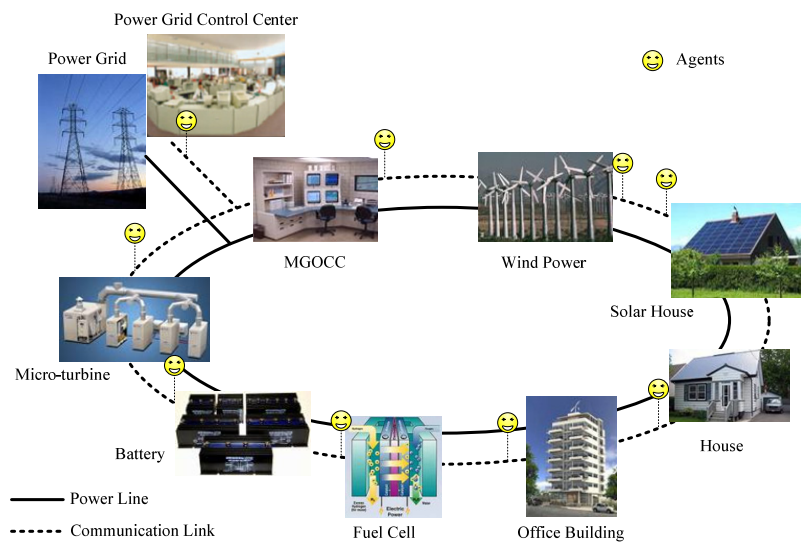

Fig. 3. Agent-based Microgrid.
The CNP is appropriately modified for microgrid operation in this study.

In the multiagent system for microgrid operation, agents take charge of facilities such as DGs, storage devices, and loads as shown in Fig. 3. Agents perceive their environment and determine their actions in order to satisfy their design purpose for microgrid operation.

\section{Design of Multiagent System}

\subsection{Basic Architecture and Interaction Among Agents}

To construct a multiagent system for microgrid operation, the following agent set $(\mathrm{Ag})$ is defined as follows:

$$
A g=\left\{A g_{M G O C C}, A G_{L}, A G_{D G}, A G_{S}, A g_{P G}\right\}
$$

where $A g_{M G O C C}$ is the MGOCC agent, $A G_{L}$ is a set of load agents $\left(A g_{L}\right), A G_{D G}$ is a set of DG agents $\left(A g_{D G}\right), A g_{S}$ is a set of storage device agents $\left(A g_{S}\right)$, and $A g_{P G}$ is a power grid agent. Fig. 4 shows the configuration of the multiagent system.

For communication among agents, a modified version of the Knowledge Query and Manipulation Language (KQML) is employed and the following message format is used:

\section{$(<$ performative $>$ :from $<$ agent name $>$ :to $<$ agent name $>$} :content $<$ OAV type data $>$ )

where OAV type data is composed of an object, an attribute of the object, and the value of the attribute.

For knowledge sharing among agents, the following template format is used:

(template name: ID identifier OAV type data list)

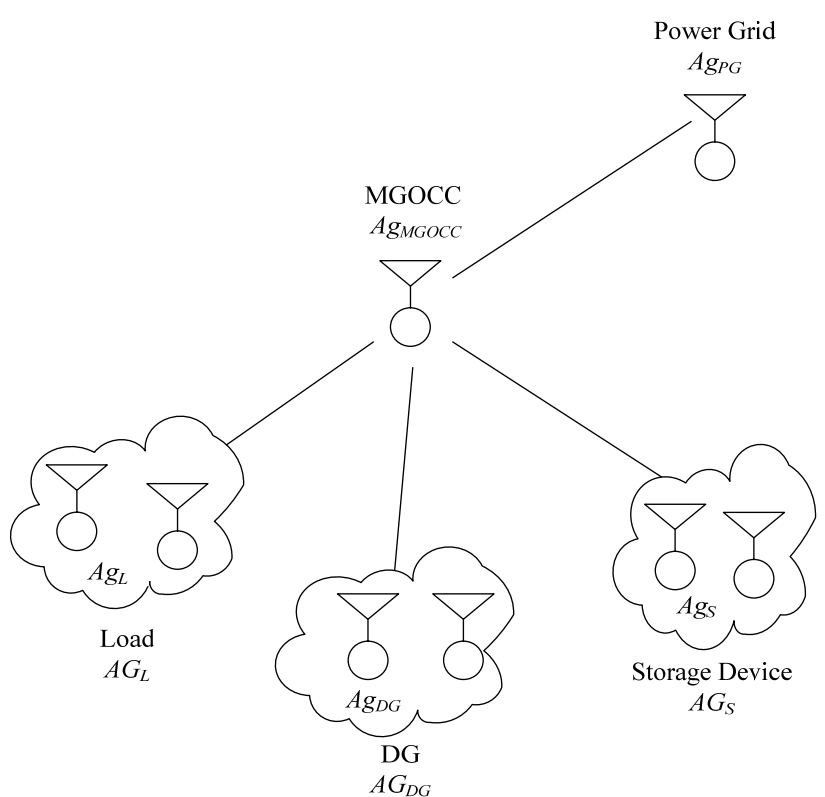

Fig. 4. Multiagent system configuration. 
The modified CNP is used as a protocol for interactions among cooperative agents. For the effective implementation of the modified CNP, the following state transition function $(T)$ is used:

$$
\left(s^{\prime}, a\right)=T(s, e)
$$

where s', a, s, and e denote the new state, the action, the current state, and the event, respectively [13].

The following procedure of the modified CNP based on market rules mentioned in section. 2.1 is applied to the multiagent system for microgrid operation. Fig. 5 shows the deadlines of the procedure.

Step 1: $A g_{M G O C C}$, as a manager, receives transaction prices $\left(P_{S}\right.$ and $\left.P_{B}\right)$ for the next interval from $A g_{P G}$.

Step 2: $A g_{M G O C C}$ announces a new task with the transaction prices via a broadcast message to every $A g_{D G}, A g_{L}$, and $A g_{S}$ by t t $_{1}$ (Fig. 5).

Step 3: $A g_{L}$ and $A g_{S}$, as consumers, inform $A g_{M G O C C}$ of their power demand by $\mathrm{t}_{2}$ (Fig. 5).

Step 4: $A g_{D G}$ and $A g_{S}$, as suppliers, evaluate the task according to

$$
\text { Bid }=\left\{\begin{array}{cc}
1 & \text { if } P_{b i d} \leq P_{B} \\
0 & \text { other }
\end{array}\right.
$$

and eligible agents submit bids by $\mathrm{t}_{2}$ (Fig. 4), where $P_{\text {bid }}$ is a bid price.

Step 5: $A g_{M G O C C}$ selects final suppliers as contractors by merit order and awards contracts to contractors by $\mathrm{t}_{3}$ (Fig. 5).

Step 6: Surplus power unselected at a price lower than or equal to $P_{S}$ is sold to the power grid and shortage power is purchased from the power grid. The transaction is carried out automatically by power flows according to physical laws and the amount is checked by a watt-hour meter after transaction is complete.

Step 7: The task is completed by submitting a report by $\mathrm{t}_{4}$ (Fig. 5) after performing the contract.

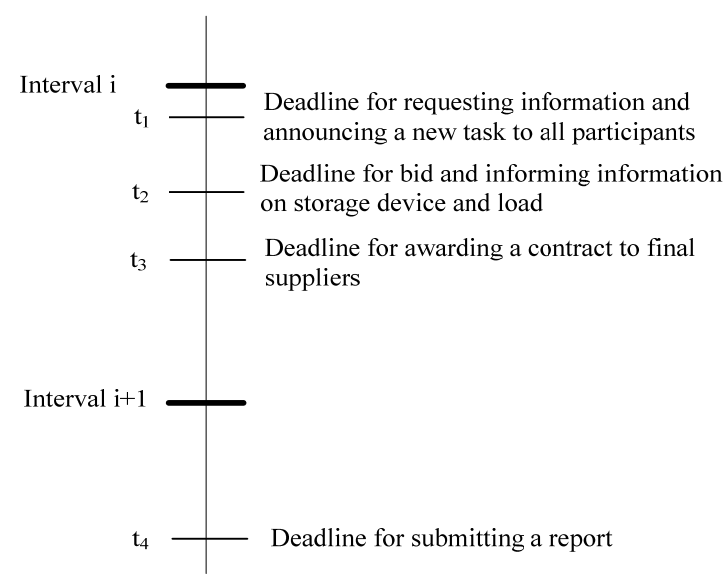

Fig. 5. Deadlines for interactions among agents.
Table 1 shows performatives for the modified CNP.

Table 1. Performatives for modified CNP

\begin{tabular}{c|c}
\hline Performative & Meaning \\
\hline AnnounceTask & Announce start of a new task \\
\hline ReceiveTask & Receive a new task \\
\hline InformLoad & Inform load amount \\
\hline Bid & Bid for power supply \\
\hline ReceiveBid & Receive a bid \\
\hline Award & Award contracts \\
\hline ReceiveAward & Receive the contract \\
\hline Report & Submit a report \\
\hline
\end{tabular}

\subsection{MGOCC Agent $\left(\boldsymbol{A g}_{M G O C C}\right)$}

$A g_{M G O C C}$ has sufficient knowledge and information to solve problems as a manager agent of the modified CNP. $A g_{M G O C C}$ has tasks such as announcing a new task, gathering information, selecting final suppliers, distributing operation results, and receiving reports from contractors. Fig. 6 shows the problem solving flow chart of the MGOCC agent.

The tasks progress via four states, as shown in Fig. 7. The "wait-task" state is a state in which the announcement of power transaction prices from $A g_{P G}$ is awaited. The "wait-bid" state is a state that occurs after $A g_{M G O C C}$ announces a new task with the power transaction prices by $t_{1}$ (Fig. 5). In this state, bids from power suppliers are awaited until $t_{2}$ (Fig. 5). The "wait-info" state occurs after $\mathrm{Ag}_{\mathrm{MGOCC}}$ awards contracts to contractor agents by $\mathrm{t}_{3}$ (Fig. 5). In this state, reports from contractor agents are awaited until $t_{4}$ (Fig. 5). The "prep" state is a state in which a series of processes are completed. In this state, $A g_{M G O C C}$ erases its working memories and returns to the "wait-task" state again for the next task. Deadlines for interactions among agents as shown in Fig. 5 are monitored by an inner timer.

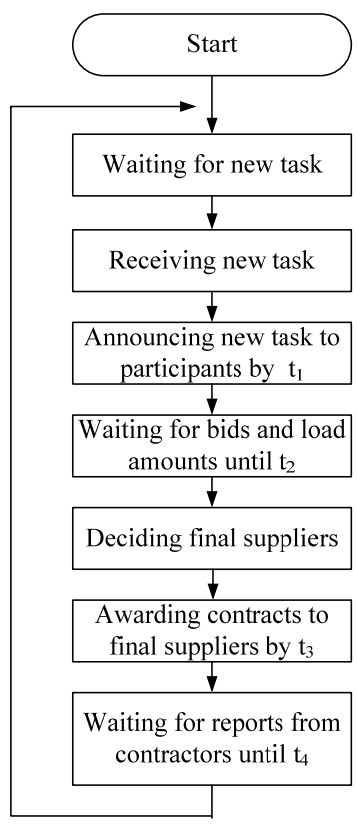

Fig. 6. Problem solving flow chart of the MGOCC agent. 


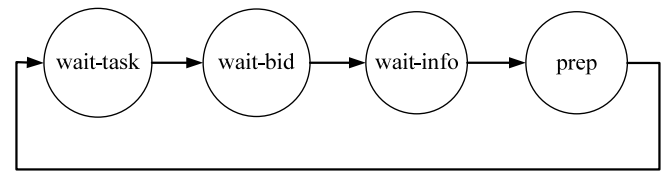

Fig. 7. State transition of the MGOCC agent.

\subsection{DG Agent $\left(A g_{D G}\right)$}

$A g_{D G}$ governs a DG or a group of identical multiple DGs located at the same place. The production output declared as a contractor is used as a desired reference for the controller of a DG or a group of identical multiple DGs. $A g_{D G}$ has the following tasks: waiting for a new task with receiving power transaction prices from $A g_{M G O C C}$ at the beginning of an interval, evaluating the task using (8), bidding for the task, waiting for the contract awarded by $A g_{M G O C C}$, receiving the contract from $A g_{M G O C C}$, and reporting the contract. Fig. 8 shows the problem solving flow chart of DG agents.

The tasks progress via three states, as shown in Fig. 9. The "wait-task" state is a state in which a new task from $A g_{M G O C C}$ is awaited. The "wait-award" is a state that occurs after $A g_{D G}$ bids by $\mathrm{t}_{2}$ (Fig. 5). In this state, the contract awarded by $A g_{M G O C C}$ by $\mathrm{t}_{3}$ (Fig. 5) is awaited. Here, $A g_{D G}$ receives the award message only if it is selected as a contractor. The "prep" state is a state in which a series of processes are completed. In this state, $A g_{D G}$ submits a report by $\mathrm{t}_{4}$ (Fig. 5) after performing the contract, erases its working memories, and returns to the "wait-task" state for the next task.

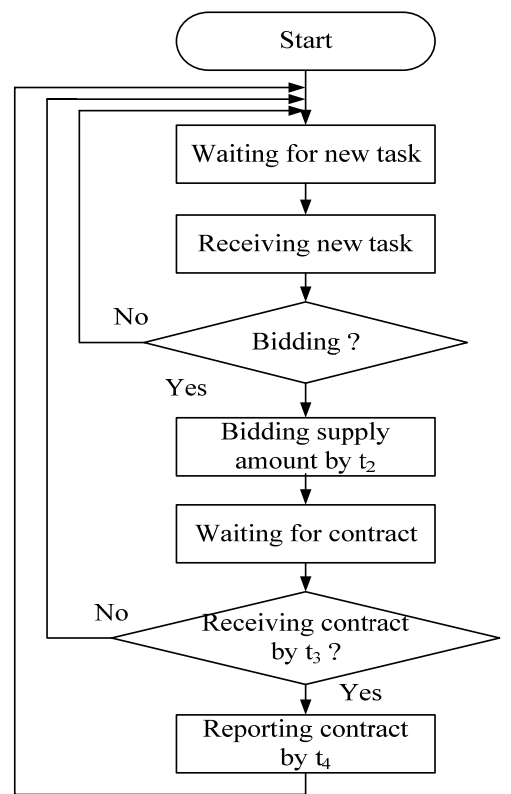

Fig. 8. Problem solving flow chart of DG agents.

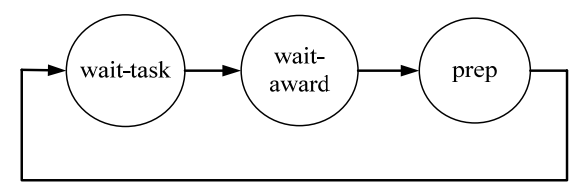

Fig. 9. State transition of DG agents.

\subsection{Load Agent $\left(\mathrm{Ag}_{L}\right)$}

$A g_{L}$ takes charge of a consumption device or a group of consumption devices located at the same place. $A g_{L}$ has the ability to forecast the amount of load for the next interval. $A g_{L}$ performs the following tasks: waiting for a new task from $A g_{M G O C C}$ at the beginning of an interval and informing the amount of its load.

The above tasks progress via two states as shown in Fig. 10. The "wait-task" state is a state in which the announcement of the beginning of a new task from $A g_{M G O C C}$ is awaited. The "prep" state is a state in which a series of processes are completed. In this state, $A g_{L}$ informs $A g_{M G O C C}$ of its load amount by $\mathrm{t}_{2}$ (Fig. 5), erases its working memories, and returns to the "wait-task" state.

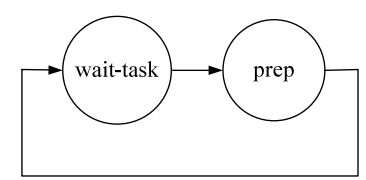

Fig. 10. State transition of load agents.

\subsection{Storage Device Agent $\left(\mathrm{Ag}_{S}\right)$}

After receiving a new task with transaction prices from $A g_{M G O C C}$ at the beginning of an interval, $A g_{S}$ chooses one of three actions: no action, charge as a consumer, and discharge as a supplier. To choose one of three actions, $A g_{S}$ has the ability to choose the action with a specific strategy. Fig. 11 shows the problem solving flow chart from storage device agents.

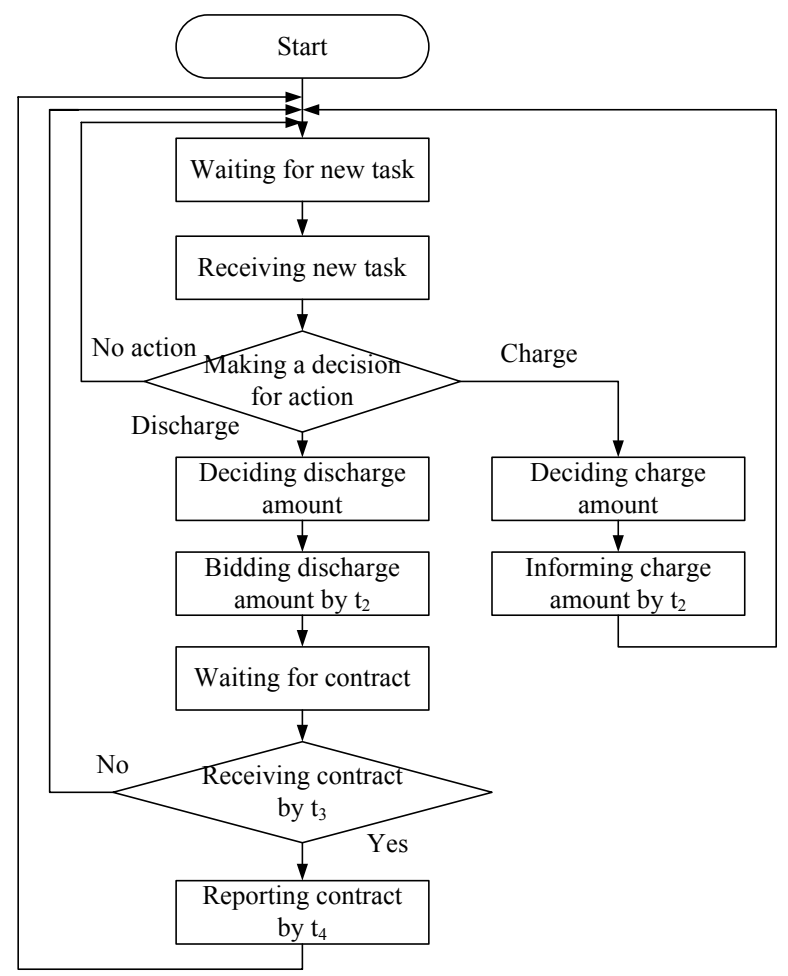

Fig. 11. Problem solving flow chart of storage device agents. 
The above tasks progress via three states shown in Fig. 9 in the case of discharge or two states shown in Fig. 10 in the case of charge.

\subsection{Power Grid Agent $\left(\boldsymbol{A g}_{P G}\right)$}

In the case of $A g_{P G}$, we consider only a function that announces power transaction prices to $A g_{M G O C C}$ at the beginning of an interval in this paper.

\subsection{Implementation}

The agent-based microgrid is designed by Distributed Agent System based on Hybrid Architecture (DASH) [14] as a multiagent platform and Interactive Design Environment for Agent Designing Framework (IDEA) as a GUIbased interactive environment for the DASH platform [15]. User defined functions are developed by Java. The agentbased microgrid is implemented under the XP window operating system. Fig. 12 shows the "receive-bid" rule of $A g_{M G O C C}$ coded on the IDEA environment as an example. In this rule, the state of $A g_{M G O C C}$ is the "wait-bid". When receiving a bid from a bidder, $A g_{M G O C C}$ calls "setData" to set the bid information to select contractor agents, where "setData" is a method of Java as a user defined function.

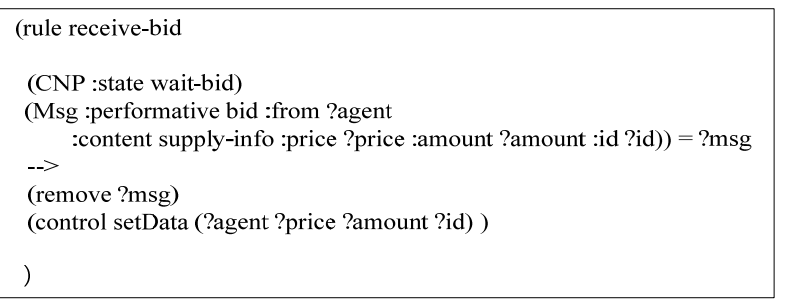

Fig. 12. Example of rule of MGOCC agent.

\section{Experiment}

To evaluate the feasibility of the proposed multiagent system for microgrid operation in the grid-interconnected mode, three experiments are carried out. The major objective of these experiments is to check whether a test microgrid is operated autonomously by the proposed multiagent system. In the case of the storage device agent, it is not easy to establish a decision-making strategy to select an action mentioned in Section 3.5 with limited information. Therefore, the storage device is not considered in these experiments.

In these experiments, a simple microgrid is used to clearly check autonomous operation of the microgrid and its design objective. The test microgrid comprises three DGs (DG1, DG2, and DG3) and a lumped load (L1) as shown in Fig. 13. Transaction prices for three experiments are listed in Table 2. For simplicity, bid prices of DGs are assumed as their production costs. Production costs of DGs and the forecasted load for the next interval are as given below:

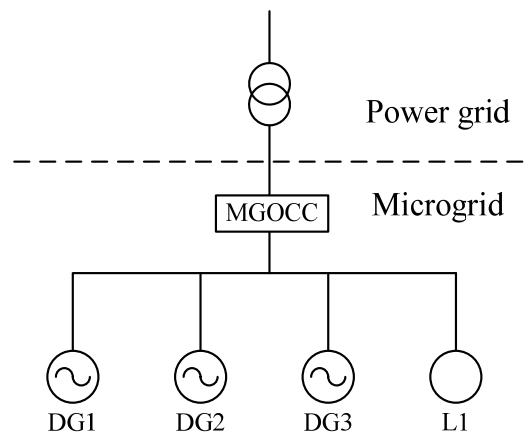

Fig. 13. Test system for Microgrid operation.

Table 2. Transaction prices

\begin{tabular}{c|c|c}
\hline Experiment & $P_{S}$ of the next interval & $P_{B}$ of the next interval \\
\hline Experiment 1 & $40 ф / \mathrm{kWh}$ & $90 ф / \mathrm{kWh}$ \\
\hline Experiment 2 & $30 \notin / \mathrm{kWh}$ & $75 ф / \mathrm{kWh}$ \\
\hline Experiment 3 & $80 ф / \mathrm{kWh}$ & $120 \notin / \mathrm{kWh}$ \\
\hline
\end{tabular}

- DG1 = production cost: $70 \notin / \mathrm{kWh}$, capacity: $10 \mathrm{kWh}$

- DG2 = production cost: $80 \phi / \mathrm{kWh}$, capacity: $25 \mathrm{kWh}$

- DG3 = production cost: $25 \phi / \mathrm{kWh}$, capacity: $5 \mathrm{kWh}$

- L1 $=30 \mathrm{kWh}$

In three experiments, the multiagent system for microgrid operation comprises six agents: an MGOCC agent $\left(A g_{M G O C C}\right)$, three DG agents $\left(A g_{D G 1}, A g_{D G 2}\right.$, and $\left.A g_{D G 3}\right)$, a load agent $\left(A g_{L I}\right)$, and a power grid agent $\left(A g_{P G}\right)$.

\subsection{Experiment 1}

Production costs of three DG3 are lower than $P_{B}$ and their aggregated production amounts are larger than the load amount in the microgrid. In this experiment, we check whether final suppliers are appropriately selected by merit order for the next interval.

Fig. 14 shows the result of Experiment 1. The result illustrates that $A g_{D G 1}, A g_{D G 2}$, and $A g_{D G 3}$ bid because their

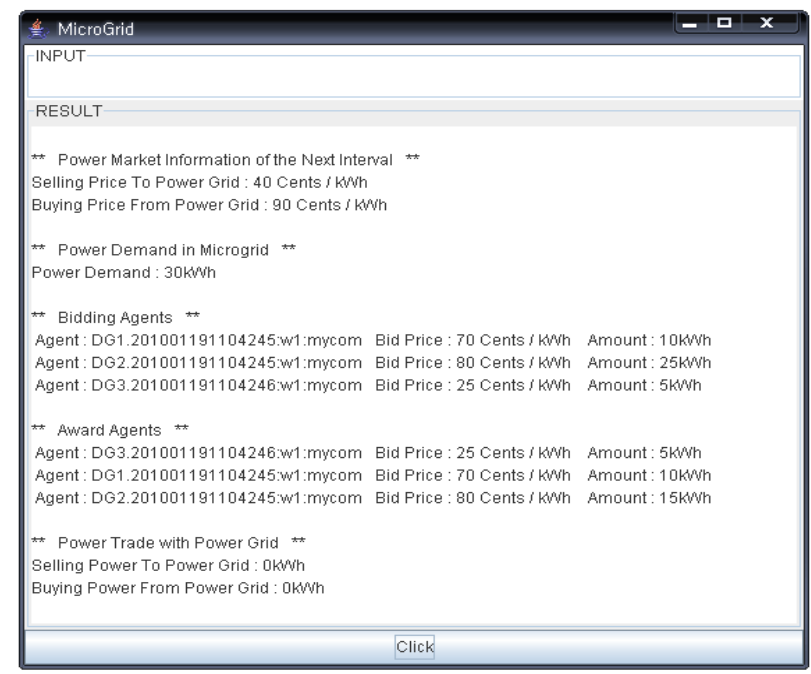

Fig. 14. Result of Experiment 1. 
production costs were lower than $P_{B}$, and they were selected as contractors by merit order. Concretely, $10 \mathrm{kWh}$ of $A g_{D G 1}, 15 \mathrm{kWh}$ of $A g_{D G 2}$, and $5 \mathrm{kWh}$ of $A g_{D G 3}$ were selected as supply power. In particular, in the case of $A g_{D G}$, only $15 \mathrm{kWh}$ of the bid amount $(25 \mathrm{kWh})$ was selected and the surplus $10 \mathrm{kWh}$ was not sold to the power grid because its bid price was higher than $P_{S}$. The result reflected well our design purpose on this operation condition.

\subsection{Experiment 2}

Production costs of DG1 and DG3 are lower than $P_{B}$ but the production cost of DG2 is higher than $P_{B}$. In this experiment, $A g_{D G I}$ and $A g_{D G 3}$ can bid and their aggregated amounts are smaller than the load amount in the microgrid. Therefore, shortage power must be purchased from the power grid. Here, we check whether the agent-based microgrid is operated satisfactorily according to our design purpose.

Fig. 15 shows the result of Experiment 2. As shown in Fig. 15, $A g_{D G 2}$ did not bid because its production cost was higher than $P_{B}$. In this experiment, $A g_{D G I}(10 \mathrm{kWh})$ and $A g_{D G 3}(5 \mathrm{kWh})$ were selected as contractors. For the power balance, it was decided to purchase $15 \mathrm{kWh}$ from the power grid for the next interval. The result reflected well our design purpose on this operation condition.

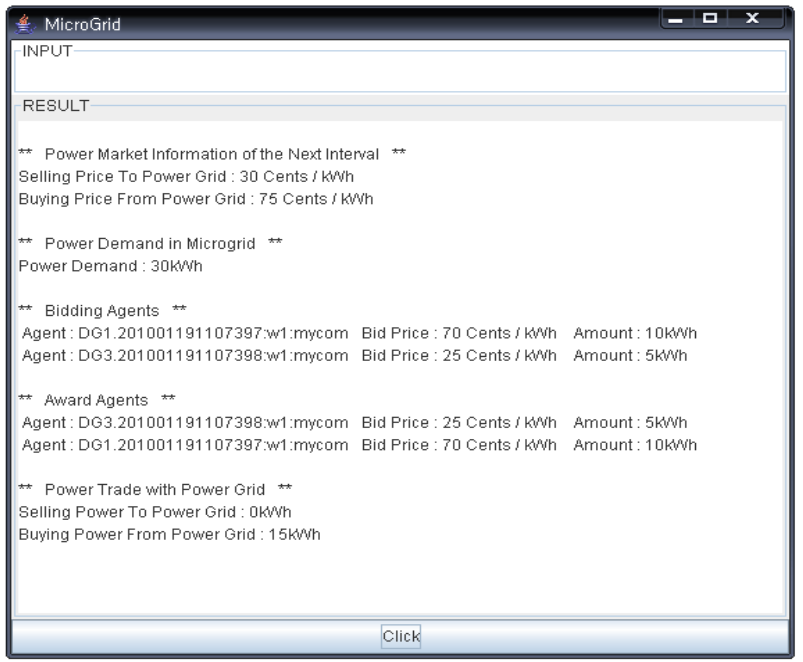

Fig. 15. Results of Experiment 2.

\subsection{Experiment 3}

Production costs of DG1 and DG3 are lower than $P_{B}$ and the production cost of DG2 is equal to $P_{B}$. In this experiment, all DG agents can bid and their aggregated amounts are larger than the load amount in the microgrid. In contrast to Experiment 1, the surplus power of DG2 can be sold to the power grid. Here, we check whether the microgrid is operated satisfactorily according to our design purpose.

Fig. 16 shows the result of Experiment 3. The result was identical to that of Experiment 1, except that $10 \mathrm{kWh}$ of $A g_{D G 2}$ unselected by merit order was sold to the power grid

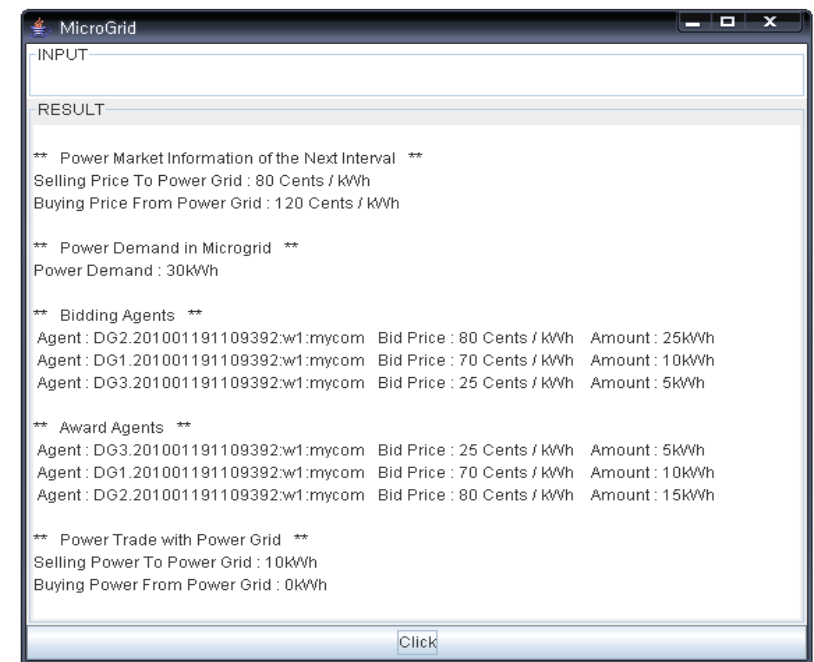

Fig. 16. Result of Experiment 3.

for the next interval because the production cost of $A g_{D G 2}$ was equal $P_{S}$. The result reflected well our design purpose on this operation condition.

\subsection{Evaluation}

We used merit order to select final suppliers and their supply amounts before power transaction with the power grid. Although merit order is a well-known optimization technique, to check the accuracy of our experiment results, we compared the results to those of linear programming (LP) using (9):

$$
\begin{array}{ll}
\text { Min } & \sum_{i} \operatorname{Cost}_{i} \cdot D G_{i}+P_{B} \cdot P_{B u y}-P_{S} \cdot P_{\text {Sell }} \\
\text { s.t. } & D G_{i 0}+D G_{i s}=D G_{i} \\
& D G_{i} \leq C a p_{i} \\
& P_{B u y} \leq M \\
& P_{\text {Sell }}=\sum_{i} D G_{i s} \\
& \sum_{i} D G_{i 0}+p_{P G}=D_{M G}
\end{array}
$$

where, $i$ : the identifier of power suppliers

Cost $t_{i}$ : the production cost of supplier $i$

$D G_{i}$ : the total power supply of the supplier $i$

$D G_{i o}$ : the power supply of the supplier i for the demand of Microgrid

$D G_{i s}$ : the power supply of the supplier i for selling power to the power grid

$P_{B}$ : the buying price from the power grid

$P_{S}$ : the selling price to the power grid

$P_{B u y}$ : power purchased from the power grid

$P_{\text {Sell }}$ : power selling to the power grid

$\mathrm{Cap}_{i}$ : the production capacity of supplier $i$

$D_{M G}$ : the demand of microgrid

$M$ : a large positive number

Table 2 shows results by LP. In Experiment 1 and Experiment 2, results by LP were equal to results by the mul- 
tiagent system. In Experiment 3, there was a relatively small difference between the LP based result and the multiagent system based result (Fig. 16). However, we can see that total profits and costs of the microgrid are the same. The reason is that the multiagent system is based on merit order and the 2-step decision on the power supply for the demand of microgrid (step 1) and the power trade to the power grid (step 2). Especially, the characteristic of merit order was shown well in Experiment 3. Through the comparison between the multiagent system based on merit order and LP we can see our experiment results are acceptable. Therefore, we conclude that the proposed multiagent system will be a good solution for microgrid operation.

Table 3. The results of linear programming

\begin{tabular}{c|c|c|c}
\hline Variables & Experiment 1 & Experiment 2 & Experiment 3 \\
\hline $\mathrm{DG}_{10}$ & $10 \mathrm{kWh}$ & $10 \mathrm{kWh}$ & $10 \mathrm{kWh}$ \\
\hline $\mathrm{DG}_{1 \mathrm{~s}}$ & - & - & - \\
\hline $\mathrm{DG}_{20}$ & $15 \mathrm{kWh}$ & - & $20 \mathrm{kWh}$ \\
\hline $\mathrm{DG}_{2 \mathrm{~s}}$ & - & - & $5 \mathrm{kWh}$ \\
\hline $\mathrm{DG}_{30}$ & $5 \mathrm{kWh}$ & $5 \mathrm{kWh}$ & - \\
\hline $\mathrm{DG}_{3 \mathrm{~s}}$ & - & - & $5 \mathrm{kWh}$ \\
\hline $\mathrm{P}_{\text {Sell }}$ & - & - & $10 \mathrm{kWh}$ \\
\hline $\mathrm{P}_{\text {Buy }}$ & - & $15 \mathrm{kWh}$ & - \\
\hline
\end{tabular}

Additionally, we expect that the following merits will be realized by using the multiagent system for microgrid operation:

- The operation costs of participants can be reduced because of saving costs for hiring operators.

- The privacy of some participants such as individual houses can be protected.

- The processing time for decision making is small because the multiagent system is based on the software and computer network.

- The multiagent can easily be applied to microgrids based on different market rules because the software-based multiagent system can be modified easily.

- The microgrid can be operated stably on unforeseen conditions because intelligent agents are inherently adaptable and hence can solve unpredicted problems.

\section{Conclusion}

In this paper, we presented a multiagent system for microgrid operation in the grid-interconnected mode. The modified CNP was used as a protocol for interactions among agents. An energy market environment with generation competition was adopted to guarantee requirements mentioned in Section 2.1. The multiagent system were designed and implemented. To verify the feasibility of the proposed multiagent system, three experiments were carried out. The results of three experiments showed that the suggested agent-based microgrid was appropriately operated on three operation conditions. The results agreed with our design purpose.
In the future work, autonomous islanded microgrid operation based on the multiagent system will be studied. Also, we plan to study a decision-making strategy for the storage device and to enhance the intelligence of agents of our multiagent system.

\section{References}

[1] N. Hatziargyriou, H. Asano, H. R. Iravani and C. Marnay, "Microgrids," IEEE Power and Energy Magazine, Vol. 5, No. 4, pp. 78-94, July-Aug. 2007.

[2] M. Barnes, J. Kondoh, H. Asano, J. Oyarzabal, G. Ventakaramanan, R. Lasseter, N. Hatziargyriou and T. Green, "Real-Word MicroGrids - An Overview," 2007 International Conference of System of Systems Engineering, pp. 1-8, 2007.

[3] P. Schavemaker and L. Sluis, Electrical Power System Essentials, John Willey and Sons, Ltd., 2008.

[4] M. Wooldridge, An Introduction to Multiagent Systems, $2^{\text {nd }}$ Edition, A. John Wiley and Sons, Ltd., Publication, 2009.

[5] S. D. J. McArthur, S. M. Strachan and Gordon Jahn, "The Design of a Multi-agent Transformer Condition Monitoring System," IEEE Trans. on Power System, Vol. 19, No. 4, pp. 1845-1852, Nov. 2004.

[6] J. M. Solanki, S. Khushalani and N. N. Schulz, “A Multi-agent Solution to Distribution Systems Restoration," IEEE Trans. on Power Systems, Vol. 22, No. 3, pp. 1026-1034, Aug. 2007.

[7] M. E. Baran and I. M. El-Markabi, "A Multiagentbased Dispatching Scheme for Distributed Generators for Voltage Support on Distribution Feeders," IEEE Trans. on Power Systems, Vol. 22, No. 1, pp. 52-59, Feb. 2007.

[8] A. L. Dimeas and N. D. Hatziargyriou, "Operation of a Multiagent System for Microgrid Control," IEEE Trans. on Power Systems, Vol. 20, No. 3, pp. $1447-$ 1455, Aug. 2005.

[9] R. G. Smith, "The Contract Net Protocol: High-level Communication and Control in a Distributed Problem Solver," IEEE Trans. on Computer, Vol. C-29, No. 12, pp. 1104-1113, Dec. 1980.

[10] G. B. Sheble, "Real-time Economic Dispatch and Reserve Allocation Using Merit Order Loading and Linear Programming Rules," IEEE Trans. on Power Systems, Vol. 4, No. 4, pp. 1414-1420, Oct. 1989.

[11] H.-M. Kim and T. Kinoshita, "Multiagent System for Microgrid Operation based on Power Market Environment," Proc. INTELEC 2009, Incheon, Korea, 2009.

[12] G Weiss, editor, Multiagent Systems: A Modern Approach to Distributed Artificial Intelligence, The MIT press, 1999.

[13] T. Kinoshita, Building Agent-based Systems, The Institute of the Information and Systems Society, Japan, 2001 (in Japanese).

[14] T. Kinoshita and K. Sugawara, “ADIPS Framework 
for Flexible Distributed Systems," T. Ishide (ed.), Multiagent Platforms, LNAI 1599, pp. 18-32, 1998.

[15] T. Uchiya, T. Maemura, L. Xiaolu and T. Kinoshita, "Design and Implementation of Interactive Design Environment of Agent System," Proc. of $20^{\text {th }}$ Int. Conf. Industrial, Engineering and Other Applications of Applied Intelligent Systems (IEA/AIE 2007), LNAI4570, AAAI/ACM, pp. 1088-1097, 2007.

[16] IDEA/DASH Tutorial [Online]. Available:

http://www.ka.riec.tohoku. ac.jp/idea/index.html.

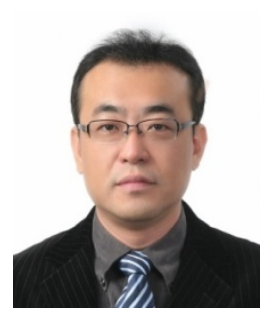

Hak-Man Kim received his B.S., M.S. and Ph.D. degrees in Electrical Engineering from Sungkyunkwan University, Korea, in 1991, 1993, and 1998, respectively. He was a senior researcher of the Korea Electrotechnology Research Institute from Oct. 1996 to Feb. 2008. Currently, he is a professor in the Department of Electrical Engineering, University of Incheon, Korea. Also, he is pursuing a Ph.D. degree from Graduate School of Information Science at Tohoku University, Japan. His research interests include power system modeling \& analysis and autonomous power system based on multiagent system.

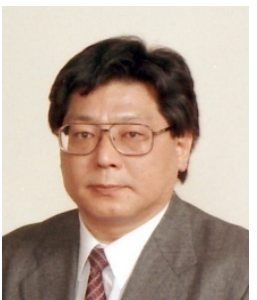

Tetsuo Kinoshita received his Dr. Eng. degree in Information Engineering from Tohoku University, Japan, in 1993. Currently, he is a professor of Graduate School of Information Science, Tohoku University, Japan. His research interests include agent engineering, knowledge engineering, and knowledge-based systems.

Dr. Kinoshita received the IPSJ Research Award, the IPSJ Best Paper Award and the IEICE Achievement Award in 1989, 1997, and 2001, respectively. He is a Fellow of IEICE and a member of IEEE, ACM, AAAI, IPSJ, JSAI, and Society for Cognitive Science of Japan. 